\title{
CIRSE Vascular Closure Device Registry
}

\author{
Jim A. Reekers • Stefan Müller-Hülsbeck • Martin Libicher • \\ Eli Atar · Jens Trentmann - Pierre Goffette $\cdot$ Jan Borggrefe $\cdot$ \\ Kamil Zeleňák $\cdot$ Pieter Hooijboer · Anna-Maria Belli
}

Received: 1 February 2010/Accepted: 12 March 2010/Published online: 28 October 2010

(C) The Author(s) 2010. This article is published with open access at Springerlink.com

\begin{abstract}
Purpose Vascular closure devices are routinely used after many vascular interventional radiology procedures. However, there have been no major multicenter studies to assess the safety and effectiveness of the routine use of closure devices in interventional radiology.
\end{abstract}

\section{J. A. Reekers ( $\square)$}

Department of Radiology, Academic Medical Center, Meibergdreef 9, NH 1105 AZ Amsterdam, The Netherlands e-mail: j.a.reekers@amc.uva.nl

\section{S. Müller-Hülsbeck}

Department of Radiology, Academic Hospitals, Flensburg, Germany

M. Libicher

Department of Radiology, University Clinics, Colonge, Germany

E. Atar

Department of Radiology, Hasharon Hospital, Petah Tikva, Israel

J. Trentmann $\cdot$ J. Borggrefe

Department of Radiology, University Clinics

Schleswig-Holstein, Kiel, Germany

P. Goffette

Department of Radiology, University Clinics St. Luc, Brussels, Belgium

\section{K. Zeleňák}

Department of Radiology, University Hospital, Martin, Slovakia

P. Hooijboer

Department of Radiology, Scheper Ziekenhuis, Emmen,

Netherlands

\author{
A.-M. Belli \\ Department of Radiology, St. Georges Hospital, London, UK
}

Methods The CIRSE registry of closure devices with an anchor and a plug started in January 2009 and ended in August 2009. A total of 1,107 patients were included in the registry.

Results Deployment success was 97.2\%. Deployment failure specified to access type was 8.8\% [95\% confidence interval (95\% CI) 5.0-14.5] for antegrade access and 1.8\% (95\% CI 1.1-2.9) for retrograde access $(P=0.001)$. There was no difference in deployment failure related to local PVD at the access site. Calcification was a reason for deployment failure in only $<0.5 \%$ of patients. Postdeployment bleeding occurred in $6.4 \%$, and most these (51.5\%) could be managed with light manual compression. During follow-up, other device-related complications were reported in $1.3 \%$ : seven false aneurysms, three hematoma $>5.9 \mathrm{~cm}$, and two vessel occlusions.

Conclusion The conclusion of this registry of closure devices with an anchor and a plug is that the use of this device in interventional radiology procedures is safe, with a low incidence of serious access site complications. There seems to be no difference in complications between antegrade and retrograde access and other parameters.

Keywords Interventional radiology - Closure device . Puncture site

\section{Introduction}

Currently, vascular closure devices are routinely used after many vascular interventional radiology procedures. In cardiology, many large studies on safety and effectiveness have been published, where reduction in procedure time and early ambulation and discharge were the main endpoints. However, there have been no major multicenter 
studies to assess the safety and effectiveness of the routine use of closure devices in interventional radiology. Only single-center data have been published [1-4]. This lack of data, combined with the popularity of closure device complications at morbidity and mortality conferences and in case reports, might give a biased impression about closure devices $[5,6]$. If their safety is to be accurately assessed, it is necessary to ascertain the number of all closure devices used in a given institute in relation to the rate of complications. To obtain these data, CIRSE conducted a registry to investigate the safety of closure devices. Although the initial registry was intended to be open to all CE-marked closure devices, only closure devices with an anchor and plug were finally included.

\section{Registry Design}

The registry was advertised through CIRSE e-mail and on the CIRSE Web site. It was open to all centers with more than 50 closure devices experience. The registry was conducted online and was hosted on an external server. The design of the registry, data management, and final reporting were performed by CIRSE. Device companies were not involved in this process. The registry was designed to collect data from up to 100 sites by members of CIRSE and intended to recruit 1,000 patients. Collected data included deployment success, time to hemostasis, time to mobilization, and time to discharge. A record of all in-hospital access site complications was also made. Patients returning with late closure device problems could also be included in this registry.

\section{Material and Methods}

The registry started in January 2009 and ended in August 2009. Twenty-eight centers from 10 European countries participated in the registry. Only acute procedure-related data were entered, and no direct patient identifiable information was obtained; there were no privacy issues. The medical ethical commission in one larger center looked at the protocol and concluded that no informed consent was needed. Only devices with an anchor and extravascular plug (Angio-Seal, St. Jude Medical) were included in the registry. A minimum experience of 50 closure devices was required to enter patients onto the registry. The contributors to the registry were asked to include consecutive patients. No in-or exclusion criteria on the indication for closure device use were given. Complications were registered before discharge and in follow-up; only complications in patients who returned spontaneously with a puncture site-related problem were registered. These were not linked to the original procedure for privacy reasons.

All $\chi^{2}$ tests we performed for statistical analysis used were standard tests.

\section{Results}

A total of 1,107 patients were included in the registry. The number of included patients per country varied between 438 and 16. Lowest inclusion per center was 1 and highest inclusion per center was 172. Of the included patients, 676 were male. Mean age distribution was between 55 and 75 years, with no difference between male and female patients. A total of $80.4 \%$ of all patients were on some form of antithrombotic medication. A total of $44.3 \%$ were receiving aspirin, $57.7 \%$ unfractionated heparin, and $10.7 \%$ clopidogrel. A total of $84.7 \%$ of all procedures were performed with retrograde arterial access and $15.3 \%$ with antegrade access. A total of $34.4 \%$ [95\% confidence interval (95\% CI) 27.2-42.2] of all patients with antegrade access had diabetes. For retrograde punctures, this was 22.5\% (95\% CI 19.8-25.4). This difference was highly significant, with a $P$-value of 0.002 . There was no difference in type of access between male and female patients. The percentage of obese patients (body mass index of $>25$ ) was equal in antegrade and retrograde access, with 23.9 and $25.1 \%$, respectively.

Deployment success was $97.2 \%$. Failures were due to calcifications $(n=5)$, device problems $(n=7)$, and other unspecified $(n=16)$. Deployment failure specified to access type was $8.8 \%$ (95\% CI 5.0-14.5) for antegrade access and $1.8 \%$ (95\% CI 1.1-2.9) for retrograde access $(P=0.001)$. There was no difference in deployment failure related to sheath size or French size of the closure device $(6 \mathrm{~F}$ or $8 \mathrm{~F})$. Nor was deployment success related to local PVD at the access site. Calcification was a reason for deployment failure in only $<0.5 \%$ of patients.

Postdeployment bleeding occurred in $6.4 \%$, and most of these $(51.5 \%)$ could be managed with light manual compression. Pressure dressing was only applied in $22.1 \%$. In $71.6 \%$ of the pressure dressing population, the pressure was applied for $<1 \mathrm{~h}$.

There were 26 reported complications, of which 13 $(1.2 \%)$ were reported as serious: two false aneurysms, six hematoma $>5.9 \mathrm{~cm}, 1$ significant bleeding, two vessel occlusion, and two vessel dissections. Access site complications were generally equal for antegrade and retrograde access, at $4 \%(95 \%$ CI $1.6-8.8)$ and $2.3 \%$ (95\% CI 1.4-3.6), respectively. This difference was not significant $(P=0.342)$. 
Time to ambulation was more than $1 \mathrm{~h}$ in $94.8 \%$ of patients. A total of $43.7 \%$ of all patients were discharged between 9 and $24 \mathrm{~h}$ after the procedure, and $52.3 \%$ were discharged after more than $24 \mathrm{~h}$. The closure device only shortened the anticipated discharge time in $12.2 \%$ of patients. Discharge was delayed after closure device in $0.9 \%$.

During follow-up, other device-related complications were reported in $1.3 \%(n=15)$ : seven false aneurysms, three hematoma $>5.9 \mathrm{~cm}$, two vessel occlusions, one partial occlusion of the vessel, and two severe pain at the access site.

We have looked at the data concerning complications and could not find a difference between high and low inclusion centers. However, the number of complications is too small to draw any scientific conclusions.

\section{Discussion}

The current negative view of the use of closure devices in peripheral vascular disease has been heavily influenced by the anecdotal reporting of complications. These complications should be viewed in the context of the overall usage of these devices in interventional practice to get a true incidence of these complications. To get a better understanding of the safety and the possible advantages of routine use of these devices after an interventional radiology procedure, CIRSE embarked on an independent closure device registry, which included more than 1,100 patients over a period of 7 months, demonstrating that these devices are widely used in interventional radiology. Most devices $(84.7 \%)$ are still used after retrograde access, probably including many over the bifurcation procedures. Almost all patients in this registry $(>80 \%)$ were receiving some form of antithrombotic therapy, which implies that the devices were used after some form of vascular intervention. Antegrade access is more commonly used in patients with diabetes, where below-the-knee pathology can be more easily reached than through an over-the-bifurcation procedure.

Deployment success was $>97 \%$. There is a significant difference in deployment success, with more failures in the antegrade access group: $8.8 \%$ in the antegrade and $1.8 \%$ in the retrograde group. Other parameters such as obesity, sheath size, and local calcifications did not seem to be predictors for deployment failure. The latter is in contradiction to the instructions for use by the manufacturer of the device. Access site complications were equal in both antegrade and retrograde access. Minor postdeployment bleeding was seen in $6.4 \%$ and was easily handled. This was probably due to the mismatch between anchor and plug, which can be seen after a puncture has been too steep.
There were $1.2 \%$ serious complications before discharge, with no difference between antegrade or retrograde access. There is one small prospective randomized trial that compares manual compression and Angio-Seal in day-care patients with peripheral interventional procedures [7]. In this study, no significant differences in complications were found between manual compression and Angio-Seal. There were no serious complications in either group, but there was a $12 \%$ failure rate in deployment of the closure device. However, there was a significant difference in time to hemostasis to the advantage of a closure device. The $1.2 \%$ puncture site complications are low. One of the main promotional reasons for the use of a closure device after an arterial intervention in interventional radiology is early ambulation with early discharge. However, even though the closure device seems to be safe and effective, less than $50 \%$ of all patients were discharged within $24 \mathrm{~h}$. This is probably more a reflection of local reimbursement policy than of concerns about the closure device, because the other $50 \%$ was discharged at between 9 and $24 \mathrm{~h}$. After discharge, a $1.3 \%$ incidence of serious complications can still be expected at the access site, and patients should be informed of this possibility.

In general, the conclusion of this registry of closure devices with an anchor and a plug is that the use of this device in interventional radiology procedures is safe, with a low incidence of serious access site complications. There seems to be no difference in complications between antegrade and retrograde access and other parameters. These results are also supported by some small and large singlecenter publications [1-4, 8, 9]. There was early ambulation, but shortening of anticipated discharge could not be proven, most likely due to external and local circumstances. Although not part of this registry, as a result of the design as an acute registration, patient comfort should also be an important issue in the decision to use a closure device after an interventional radiology procedure.

Conflict of interest The authors declare that they have no conflict of interest.

Open Access This article is distributed under the terms of the Creative Commons Attribution Noncommercial License which permits any noncommercial use, distribution, and reproduction in any medium, provided the original author(s) and source are credited.

\section{References}

1. Kirchhof C, Schickel S, Schmidt-Lucke C, Schmidt-Lucke JA (2002) Local vascular complications after use of the hemostatic puncture closure device Angio-Seal. Vasa 31:101-106

2. Kapoor B, Panu A, Berscheid B (2007) Angio-seal in antegrade endovascular interventions: technical success and complications in a 55-patient series. Endovasc Ther 14:382-386 
3. Kadner A, Schmidli J, Schwegler I et al (2008) Complications associated with the arterial puncture closure device-Angio-Seal. Vasc Endovasc Surg 42:225-227

4. Looby S, Keeling AN, McErlean A et al (2008) Efficacy and safety of the angioseal vascular closure device post antegrade puncture. Cardiovasc Intervent Radiol 31:558-562

5. Eidt JF, Habibipour S, Saucedo JF et al (1999) Surgical complications from hemostatic puncture closure devices. Am J Surg 178:511-516

6. van der Steeg HJ, Berger P, Krasznai AG et al (2009) Acute arterial occlusion after deployment of the Angio-Seal closure device: is it as uncommon as we think? Eur J Vasc Endovasc Surg 38:715-717
7. Upponi SS, Ganeshan AG, Warakaulle DR et al (2007) Angioseal versus manual compression for haemostasis following peripheral vascular diagnostic and interventional procedures - a randomized controlled trial. Eur J Radiol 61:332-334

8. Katzenschlager R, Tischler R, Kalchhauser G et al (2009) AngioSeal use in patients with peripheral arterial disease (ASPIRE). Angiology 60:536-538

9. Mukhopadhyay K, Puckett MA, Roobottom CA (2005) Efficacy and complications of Angioseal in antegrade puncture. Eur $\mathbf{J}$ Radiol 56:409-412 